\title{
Laparoscopic cecostomy tube placement in an Indiana pouch
}

\author{
Scott E. Delacroix Jr, MD; ${ }^{*}$ Richard Vanlangendonck, MD; ${ }^{*}$ J. Christian Winters, MD ${ }^{* \dagger}$
}

\begin{abstract}
A cecostomy tube is normally placed in an Indiana pouch for drainage and irrigation in the postoperative period. A clinical dilemma occurs when the cecostomy tube fails or is dislodged in the early postoperative period. We present the laparoscopic replacement of a cecostomy tube in the immediate postoperative period.
\end{abstract}

Can Urol Assoc J 2010;4(1):E7-E8

\section{Introduction}

During the creation of a continent cutaneous reservoir, such as the Indiana pouch, a small calibre catheter is usually placed through the imbricated efferent limb of ileum for 48 hours. ${ }^{1}$ It is normally removed in the early postoperative period. A large calibre mallencott drainage tube is usually placed in the reservoir (normally through the cecum) at the time of surgery and used for irrigation and maximal drainage to facilitate reservoir healing. This large catheter is usually removed as an outpatient procedure 2 to 3 weeks postoperatively. Prior to removal, it is clamped and patients are instructed on the catheterization of the efferent limb. Thus, early maintenance of the drainage is vitally important. In the event of a nonfunctioning or dislodged catheter in the early postoperative period, replacement is prudent. In the pediatric population with spinal dysraphism, laparoscopic cecostomy tube placement for anterograde enemas has been described. ${ }^{2,3}$ Multiple urinary diversion procedures, such as the Studer pouch, ${ }^{4}$ rectosigmoid pouch ${ }^{5}$ and ileal conduit, ${ }^{6}$ have been performed with minimally invasive intracorporeal techniques, although catheterizable cutaneous reservoirs are normally completed extracorporeally with a small laparotomy. ${ }^{7}$ Regardless of the technique of the initial operation, proactive management options include replacement by repeat laparotomy, percutaneous placement, or, as described in this case report, laparoscopic cecostomy tube placement.

\section{Case report}

A 46-year-old paraplegic man with a neurogenic bladder resulting from a thoracic vertebral fracture after a motorcycle accident presents with a long history of incontinence, poorly compliant bladder and bilateral vesico-ureteral reflux. He was on high-dose anticholinergic therapy and compliant with intermittent catheterization, but still only had a functional bladder capacity of $50 \mathrm{~mL}$. He later developed a severe sacral decubitus, which ultimately extended to the urethra. After counselling, he elected to undergo simple cystectomy and Indiana pouch construction.

The cystectomy and Indiana pouch were completed in an open surgical fashion without complications. Twentyfour hours after the operation, the patient was being transferred to a wheelchair and the cecostomy tube was inadvertently dislodged. The patient was asymptomatic, but saline flush through the efferent limb of the Indiana pouch was not adequate for mucous removal. Having a fresh pouch with only 12 French drainage through the continent limb was a concern for the operative team because of the inherent risk of obstruction due to mucous clot. Replacement of an adequate drainage tube would help prevent sequela from mucous obstruction and allow for controlled instruction on pouch usage for the patient (checking residuals during the first postoperative weeks, while learning the stoma catheterization technique). The surgeons on this case discussed the percutaneous placement, open replacement or laparoscopic replacement. There was concern over the percutaneous approach with the risk of placing the cecostomy tube through one of the running anastamotic sutures of the reservoir and subsequent pouch/suture-line failure.

Thirty-six hours after the initial open surgery, the patient was brought to the operating room and placed in the supine position. After the induction of anesthesia, 3 optical trocars ( $5 \mathrm{~mm}$ diameter) were placed after standard veress needle insufflation. These trocars were placed in the left lower quadrant in a triangular fashion. Adhesions were lysed with blunt mobilization and the reservoir was visualized in the right lower quadrant. The prior cecostomy tube site was visualized. A small incision in the skin was made over the reservoir 


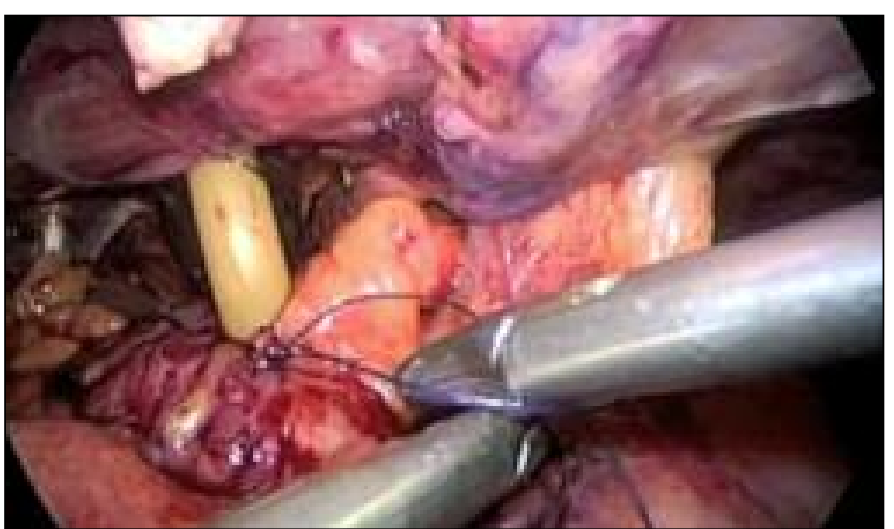

Fig. 1. Laparoscopic placement of mallencott catheter into Indiana pouch.

and a 24 French mallencott catheter was introduced into the abdominal cavity through a $12 \mathrm{~mm}$ trocar placed under direct visualization. After careful delineation of suture lines, the mallencott was placed directly into the pouch through the former cecostomy tube site (Fig. 1). A purse-string with 3-0 absorbable-braided suture was placed around the catheter. The reservoir was then affixed to the anterior abdominal wall using absorbable 2-0 suture. Operative time was 40 minutes. The patient recovered uneventfully. His 12 French efferent limb catheter was removed on postoperative day (POD) 3 and he was discharged on POD 6 with his cecostomy tube (mallencott) in place, which is in line with our usual hospital protocol for this procedure. There were no complications with this approach. It allowed for the re-establishment of optimal drainage in a controlled well-visualized fashion without the need for repeat laparotomy.

\section{Discussion}

In the early postoperative period, laparoscopic replacement of a cecostomy tube is feasible and may avoid the inherent risk of trans-suture line placement with a percutaneous approach. The replacement of the cecostomy tube allows for adequate removal of mucous and patient instruction during the first 3 weeks after surgery. Although laparoscopic cecostomy tube placement has been described previously in the pediatric and general surgical literature, ${ }^{3,8}$ this is the first report of the procedure to address a dislodged catheter in the postoperative period after open cystectomy and Indiana pouch creation.

From the ${ }^{*}$ Ochsner Clinic Foundation, Department of Urology; ${ }^{\dagger}$ Lovisiana State University Health Science Center, Department of Urology, New Orleans, LA

Competing interests: None declared.

This paper has been peer-reviewed.

\section{References}

1. Benson, MC, McKiernan JM, Olsson CA. Cutaneous Continent Urinary Diversion. In: Campbell-Walsh Urology. AJ Wein, LR Kavoussi, AC Novick, et al. (eds). Philadelphia: Saunders Elsevier, vol. 3, pp. 2586-612, 2007.

2. Yagmurlu A, Harmon CM, Georgeson KE. Laparoscopic cecostomy button placement for the management of fecal incontinence in children with Hirschsprung's disease and anorectal anomalies. Surg Endosc 2006;20:624-7.

3. Lorenzo AJ, Chait PG, Wallis MC, et al. Minimally invasive approach for treatment of urinary and fecal incontinence in selected patients with spina bifida. Urology 2007;70:568-71.

4. Gill IS, Kaouk JH, Meraney AM, et al. Laparoscopic radical cystectomy and continent orthotopic ileal neobladder performed completely intracorporeally: the initial experience. J Urol 2002;168:13-8.

5. Türk I, Deger S, Winkelmann B, et al. Laparoscopic radical cystectomy with continent urinary diversion (rectal sigmoid pouch) performed completely intracorporeally: the initial 5 cases. I Urol 2001;165(6P+1):1863-6.

6. Irwin B, Gill I, Haber G, et al. Laparoscopic Radical Cystectomy: Current Status, Outcomes, and Patient Selection. Curr Treat Options Oncol 2009; 10:243-55.

7. Ferron G, Lim TYK, Pomel C, et al. Creation of the miami pouch during laparoscopic-assisted pelvic exenteration: the initial experience. Int I Gynecol Cancer 2009;19:466-70.

8. Vaughn P, Schlinkert RT. Management of cecal perforation secondary to Ogilvie's syndrome by laparoscopic tube cecostomy. J Laparoendosc Surg 1995;5:339-41.

Correspondence: Dr. Scott E. Delacroix Jr, 1542 Tulane Ave., 5th Floor, Room 547, New Orleans, LA 70112; vrologydoc@mac.com 